\title{
(2) OPEN ACCESS \\ Making football safer for women: a systematic review and meta-analysis of injury prevention programmes in 11773 female football (soccer) players
}

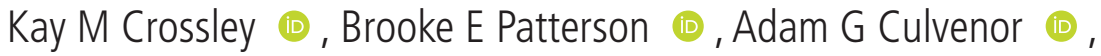

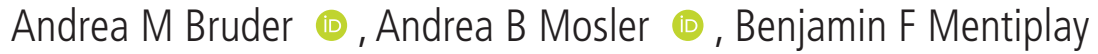

- Additional material is published online only. To view, please visit the journal online (http://dx.doi.org/10.1136/ bjsports-2019-101587).

\section{Correspondence to}

Dr Benjamin F Mentiplay, La Trobe Sport and Exercise Medicine Research Centre, La Trobe University, Melbourne, Victoria, Australia;

b.mentiplay@latrobe.edu.au

Accepted 19 March 2020 Published Online First 6 April 2020

\section{Check for updates}

(c) Author(s) (or their employer(s)) 2020. Re-use permitted under CC BY-NC. No commercial re-use. See rights and permissions. Published by BMJ.

To cite: Crossley KM, Patterson BE, Culvenor AG, et al. Br I Sports Med 2020:54:1089-1098.
ABSTRACT
Objective To evaluate the effects of injury prevention programmes on injury incidence in any women's football code; explore relationships between training components and injury risk; and report injury incidence for women's football.

Design Systematic review and meta-analysis.

Data sources Nine databases searched in August 2019.

Eligibility criteria Randomised controlled trials evaluating any injury prevention programme (eg, exercise, education, braces) were included. Study inclusion criteria were: $\geq 20$ female football players in each study arm (any age, football code or participation level) and injury incidence reporting.

Results Twelve studies, all in soccer, met inclusion criteria, with nine involving adolescent teams (aged $<18$ years). All studies (except one) had a high risk of bias. Eleven studies examined exercise-based programmes, with most $(9 / 11)$ including multiple $(\geq 2)$ training components (eg, strength, plyometric, balance exercises). Multicomponent exercise programmes reduced overall (any reported) injuries (incidence rate ratio (IRR) $0.73,95 \% \mathrm{Cl} 0.59$ to 0.91 ) and $\mathrm{ACL}$ injuries (IRR 0.55, 95\% Cl 0.32 to 0.92). For exercise-based strategies (single-component and multicomponent), hamstring injuries were also reduced (IRR 0.40, 95\% Cl 0.17 to 0.95$)$. While exercise-based strategies resulted in less knee, ankle and hip/groin injuries, and the use of multiple training components was associated with greater reductions in overall and knee injuries, further studies would be required to increase the precision of these results. The incidence of overall injuries in women's football was 3.4 per 1000 exposure hours; with ankle injuries most common.

Conclusion In women's football, there is lowlevel evidence that multicomponent, exercise-based programmes reduce overall and $\mathrm{ACL}$ injuries by $27 \%$ and $45 \%$, respectively.

PROSPERO registration number CRD42018093527.

\section{INTRODUCTION}

Women's professional, semi-professional and community football is one of the fastest growing sports worldwide. ${ }^{1-3}$ However, the known physiological, psychological and social benefits of sports participation ${ }^{45}$ can be offset by the risk of injury. In football codes, lower-limb injury (eg, knee, ankle, hip/groin, hamstring) occur frequently, ${ }^{6}{ }^{7}$ with previous injury the largest risk factor for sustaining an injury. ${ }^{8-11}$ Injury, fear of injury, lack of physical skills or strength are barriers to sport and physical activity participation in adolescent girls, ${ }^{12}$ hence the need to know whether programmes that aim to reduce injury in women's football are effective or not.

When accounting for football exposure, women have a different injury risk profile than men. The risk of serious knee injury (such as ACL rupture) is at least double in women than in men, regardless of exposure or participation level. ${ }^{13}$ Women have a higher risk of concussion, ${ }^{14}{ }^{15} \mathrm{knee}^{16}$ and ankle injuries than men, ${ }^{17}$ with men at greater risk of hamstring ${ }^{18}$ and groin injuries. ${ }^{19}$ Football codes have a higher incidence of ACL rupture and associated burden, ${ }^{20-23}$ than do other sports. ${ }^{24}$

Lower-limb injuries occur due to a dynamic interaction of multiple risk factors, ${ }^{25}$ some that may be addressed with injury prevention programmes. Many programmes have been investigated (eg, insoles or external joint supports), but mostly exercisebased strategies. ${ }^{26}$ Exercise-based injury prevention programmes typically focus on improving strength, balance and whole body biomechanics during activities similar to non-contact ACL injury mechanisms (such as changing direction or landing from a jump). ${ }^{27-29}$ Such programmes include the FIFA $11+,{ }^{30} 31$ Prevent injury and Enhance Performance programme (PEP), ${ }^{32}$ Knäkontroll ${ }^{33}$ and Footy First. $^{34}$ These exercise-based programmes include multiple training components, such as agility, balance, mobility, plyometric, running and strength activities. Systematic reviews confirm the efficacy of such programmes to reduce ACL injuries, ${ }^{35}$ for all athletes and all sports $(\mathrm{OR}=0.50 ; 95 \% \mathrm{CI} 0.41$ to $0.59)$, and female athletes specifically $(\mathrm{OR}=0.33$; $95 \%$ CI 0.27 to 0.41 ). No studies have specifically synthesised the literature for female football players, to determine the efficacy of injury prevention programmes on all injuries, and other local injuries (eg, knee, ACL, ankle, hip/groin, hamstring). Determining the sex-specific and sport-specific efficacy of injury prevention programmes will provide patients, clinicians and sporting organisations with context-specific evidence-based recommendations.

Our aim was to systematically review the effect of any type of injury prevention programme on reducing overall injury incidence in women's football. Secondary aims were to: (1) examine the effect on reducing the incidence of ACL, knee, ankle, hip/groin and hamstring injuries; (2) describe the exercise-based training components (eg, strength, balance) included in each exercise-based 
programme, and explore the relationship between the number of training components and injury incidence; and (3) report the injury incidence for lower-limb injuries for female football players using control group (ie, non-intervened) data.

\section{METHODS}

\section{Search strategy and selection criteria}

This systematic review is reported using the Preferred Reporting Items for Systematic Reviews and Meta-Analysis guidelines. ${ }^{36}$ An electronic search of nine online databases (AMED, CINAHL, Cochrane CENTRAL, EMBASE, PEDro, PubMed, Scopus, SPORTDiscus and Web of Science) was performed from inception until August 2019. The searches combined terms related to female football players, injury prevention programmes and randomised controlled trials (online supplementary appendix table 1). Specific selection criteria were: i) randomised controlled trials including any type of injury prevention programme (eg, exercise, education, braces) and a control group that was not exposed to the intervention; ii) $\geq 20$ female football players in each study arm (any age, any football code, any participation level); and iii) reported an outcome relating to injury incidence for any injury.

Studies including men and women, or studies that included participants who played sports other than football, were included if it was possible to extract the data specific to female football players. Only full-text articles published in English were included, with grey literature excluded. When eligibility could not be confirmed from the reported data, the authors were contacted for additional information. Targeted searching of the reference lists of included articles was also performed to identify any articles not found using the systematic database search strategy.

\section{Study selection, data extraction and quality assessment}

Two reviewers (BFM and AGC) independently screened all titles and abstracts of identified reports for eligibility. Full-text manuscripts of potentially relevant articles were retrieved and evaluated against the selection criteria to determine the final list of included articles.

Two reviewers (BFM and AGC) independently extracted data from included articles, with a third reviewer (ABM) consulted to resolve any conflicting results from the data extraction. Data extracted were number of participants (total and per study arm), participant characteristics (age), recruitment sources of participants and the location of the studies, details of the intervention (type, frequency, duration of sessions) and injury outcomes (number of injuries, exposure hours). For the primary aim of the current review, the number of injuries were extracted for reported 'overall injuries'. While the definition of injury differed between studies (eg, only lower-limb vs whole body, or unclear in their reporting of injuries), overall injuries refers to the total number of injuries reported in the study. The number of injuries in specific lower-limb regions were also extracted to examine ACL, knee (total knee injuries including ACL injuries), ankle, hip/groin and hamstring injuries. The results from the control groups were pooled for the secondary aim to estimate overall injury incidence, as well as for specific lower-limb regions. We did this for all female football players (injury per 1000 exposure hours) for all included studies as well as for the subset of adolescent players ( $<18$ years). For exposure data reported as athletic exposures (ie, one athlete participating in one session), the athletic exposures were converted using the assumption that one athletic exposure equalled two exposure hours, as done previously. ${ }^{37}$ Estimated exposure hours were calculated if missing data could not be provided by the authors of included studies.

Two reviewers (BEP and ABM) independently extracted data for the secondary aim relating to the exercise-based training components included in each intervention, which were categorised into one of six components (online supplementary appendix table 2). Five components (agility, balance, mobility, plyometric and strength) were adapted from previous work, ${ }^{38}$ with the addition of a running component (ie, warm-up jogging, high-speed running). Each study was classified as including a component if they described at least one activity pertaining to the component definition. Furthermore, strength and plyometric components were assessed against muscular strength and power training prescription guidelines for adults with intermediate experience ${ }^{39}$ as well as for adolescents (if average age in the study was $<18$ years) ${ }^{40}$ to determine if they met the required criteria. If studies had unclear reporting of the intervention activities, referenced work of the intervention were examined where available.

Two reviewers (ABM and $\mathrm{AMB}$ ) independently assessed the methodological quality of included studies using the Cochrane Collaboration Risk of Bias Tool, V.1. ${ }^{41}$ A third reviewer (KMC) was consulted to resolve any discrepancies in scoring. For each included study, the seven domains of the tool were rated as low, unclear or high risk of bias. Cohen's kappa was calculated to assess the extent of agreement between reviewers for the seven domains, where a kappa of $0.61-0.80$ was deemed a substantial level of agreement and $\geq 0.80$ as almost perfect. ${ }^{42}$ Based on the Cochrane recommendations, ${ }^{41}$ each study was then rated overall as low (all domains low risk), unclear (all domains low or unclear risk) or high risk of bias (one or more domains high risk). Any discrepancies for study selection, data extraction and quality assessment were initially resolved through discussion and mutual consensus, with a third reviewer consulted if consensus could not be reached.

\section{Quantitative synthesis}

Where methodological homogeneity allowed, data were pooled for meta-analysis. To examine the effects of the injury prevention programmes on overall injury rates, the pooled overall injury incidence rate ratio (IRR) and 95\% CIs were calculated using random-effects meta-analysis. This analysis was repeated for injuries relating to specific body regions, including ACL, knee, ankle, hip/groin and hamstring injuries. For the meta-analyses in exercise-based programmes, studies that incorporated multiple exercise-based training components in their intervention group were examined as a subgroup, along with an overall pooled estimate for all included studies (including both multiple and single exercise-based training components studies together). Stata V.15.1 (StataCorp, Texas, USA) was used to perform the meta-analyses and calculate the pooled estimates, with forest plots generated to allow a visual representation of each study and the pooled estimate. Between-study statistical heterogeneity was evaluated for each outcome using the standard $\mathrm{I}^{2}$ statistic (ie, the percentage of variability in prevalence estimates that is due to heterogeneity rather than chance, $0 \%=$ no inconsistency, $100 \%=$ maximal inconsistency), ${ }^{43}$ with a significance threshold of $p<0.05$. For the secondary aim, meta-regression plots were generated to examine the relationship between the number of exercise-based training components included in the interventions and the IRR. The number of training components were added together for each study, with a highest possible score of 6 if the intervention included all six training components (1 point 
for included, 0 points if not, 0.5 points when either strength or plyometric components did not meet specific training prescription guidelines). The meta-regression plots were generated if five or more studies were able to be pooled. Examination of tau squared $\left(\tau^{2}\right)$ was used to detect a potential ability of the covariate (number of training components) to explain the heterogeneity in the IRR.

The Grades of Recommendation, Assessment, Development and Evaluation (GRADE) approach was also used to evaluate the quality of evidence in each meta-analysis. ${ }^{44}$ As our inclusion criteria meant that all studies were randomised controlled trials, each meta-analysis was considered the highest level of evidence (levels of quality were very low, low, moderate or high); however, this rating was downgraded based on predetermined criteria: i) the risk of bias rating was high for most studies in the meta-analysis (risk of bias); ii) there was greater than low levels of statistical heterogeneity between studies as indicated by an $\mathrm{I}^{2}>40 \%$ (inconsistency) ${ }^{45}$; iii) the meta-analysis contained participants from varied football codes or interventions such as exercise and education (indirectness); iv) the clinical course of action would change if the upper versus lower CIs represented the truth ${ }^{46}$; v) there were large CIs around the pooled estimate of the IRR as indicated by the upper and lower CIs having $>0.50$ difference (imprecision) ${ }^{45}$; vi) the majority of studies were commercially funded; or vii) there was asymmetry of a funnel plot if $>12$ trials were included in the meta-analysis (publication bias). ${ }^{47} 48$

\section{RESULTS}

\section{Search results}

From an initial yield of 2074 articles, 12 studies were included in this review (figure 1). ${ }^{49-60}$ All studies examined soccer, with no studies identified that included other football codes.

\section{Quality assessment}

The risk of bias study ratings are presented in table 1 , and individual domain ratings for each study are reported in online supplementary appendix table 3 . All studies, except one, ${ }^{50}$ received an overall rating of high risk of bias. Cohen's kappa was 0.72 (95\% CI 0.59 to 0.85$)$, indicating substantial agreement between reviewers.

\section{Study characteristics}

Sample size of included studies ranged from 43 to 4564 analysed participants (table 1). Seven studies were conducted in Europe, ${ }^{5155-60}$ and five studies in North America. ${ }^{49} 50{ }^{52-54}$ Most studies (9/12) involved adolescent teams ( $<18$ years), with six

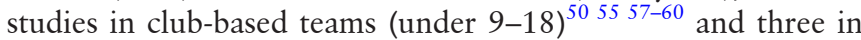
schools. ${ }^{495354}$ One study was in college teams (average age of

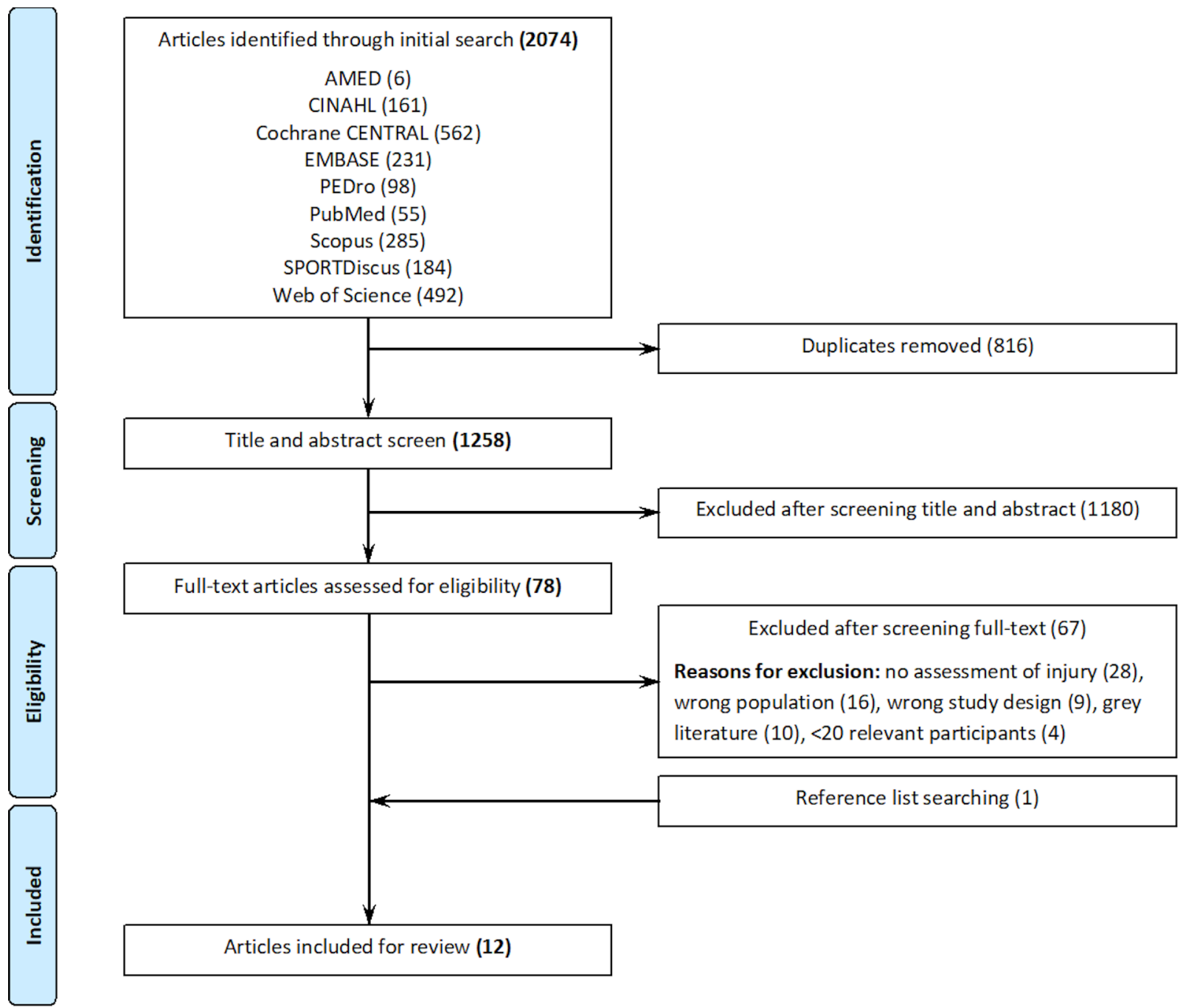

Figure 1 Flow chart of study selection. 


\begin{tabular}{|c|c|c|c|c|c|c|c|}
\hline Study & Country & $\begin{array}{l}\text { Population } \\
\text { (all soccer) }\end{array}$ & Age & Intervention & Intervention details* & $\begin{array}{l}\text { Sample size } \\
\text { analysedt }\end{array}$ & Risk of bias \\
\hline Barber Foss et a ${ }^{49}$ & USA & Middle and high schools & $14.0 \pm 1.7 \ddagger$ & $\begin{array}{l}\text { Neuromuscular training } \\
\text { (CORE intervention) }\end{array}$ & $\begin{array}{l}3 \times \text { week preseason }(20- \\
25 \mathrm{~min}) \\
2 \times \text { week in-season }(10-15 \mathrm{~min})\end{array}$ & $\begin{array}{l}\text { Total: } 142 \\
\text { IG: } 74 \\
\text { CG: } 68\end{array}$ & High \\
\hline Emery and Meeuwisse $\mathrm{e}^{50}$ & Canada & $\begin{array}{l}\text { Under-13 to } 18 \text { teams } \\
\text { (indoor soccer) }\end{array}$ & $\begin{array}{l}\text { IG: U13-15: 54\% } \\
\text { IG: U16-18: } 46 \% \\
\text { CG: U13-15: } 38.6 \% \\
\text { CG: U16-18: } 61.4 \%\end{array}$ & $\begin{array}{l}\text { Neuromuscular training } \\
\text { (plus home-based balance } \\
\text { training) }\end{array}$ & $\begin{array}{l}\text { Every practice and game } \\
(15 \mathrm{~min}) \\
\text { Balance board at least } 3 \times \\
\text { week ( } 15 \mathrm{~min})\end{array}$ & $\begin{array}{l}\text { Total: } 412 \\
\text { IG: } 161 \\
\text { CG: } 251\end{array}$ & Unclear \\
\hline Espinosa et $a^{51}$ & Spain & $\begin{array}{l}\text { First and second National } \\
\text { divisions }\end{array}$ & $\begin{array}{l}\text { Team } 1: 23 \pm 4 \\
\text { Team 2: } 19 \pm 2 \\
\text { Team 3: } 22 \pm 3\end{array}$ & Eccentric hamstring exercises & $\begin{array}{l}2 \times \text { week (estimated } \\
10-15 \mathrm{~min})\end{array}$ & $\begin{array}{l}\text { Total: } 43 \\
\text { IG: } 22 \\
\text { CG: } 21\end{array}$ & High \\
\hline Gilchrist et $a^{52}$ & USA & College teams (NCAA) & $\begin{array}{l}\text { IG: } 19.88 \\
\text { CG: } 19.88\end{array}$ & $\begin{array}{l}\text { Neuromuscular training (PEP } \\
\text { programme) }\end{array}$ & $3 \times$ week (<30 min) & $\begin{array}{l}\text { Total: } 1435 \\
\text { IG: } 583 \\
\text { CG: } 852\end{array}$ & High \\
\hline Heidt et $a l^{53}$ & USA & High schools & $14-18$ & $\begin{array}{l}\text { Neuromuscular training } \\
\text { (Frappier Acceleration } \\
\text { Training programme) }\end{array}$ & $\begin{array}{l}3 \times \text { week during preseason only } \\
\text { (unknown duration) }\end{array}$ & $\begin{array}{l}\text { Total: } 300 \\
\text { IG: } 42 \\
\text { CG: } 258\end{array}$ & High \\
\hline LaBella et a $\left.\right|^{54}$ & USA & High schools & $\begin{array}{l}\text { IG: } 16.52 \\
\text { CG: } 16.37\end{array}$ & Neuromuscular training & $\begin{array}{l}\text { Every practice }(20 \mathrm{~min}) \text { and } \\
\text { game (shorter version) }\end{array}$ & $\begin{array}{l}\text { Total: } 654 \\
\text { IG: } 321 \\
\text { CG: } 333\end{array}$ & High \\
\hline Rössler et a $\left.\right|^{55}$ & $\begin{array}{l}\text { Switzerland, } \\
\text { Germany, Czech } \\
\text { Republic, The } \\
\text { Netherlands }\end{array}$ & Under-9 to 13 teams & $\begin{array}{l}\text { IG: } 11.6 \pm 1.1 \\
\text { CG: } 11.4 \pm 1.0\end{array}$ & $\begin{array}{l}\text { Neuromuscular training (FIFA } \\
11+\text { Kids) }\end{array}$ & At least $2 \times$ week $(15-20 \mathrm{~min})$ & $\begin{array}{l}\text { Total: } 171 \\
\text { IG: } 103 \\
\text { CG: } 68\end{array}$ & High \\
\hline Söderman et al ${ }^{56}$ & Sweden & $\begin{array}{l}\text { Second and third } \\
\text { National divisions }\end{array}$ & $\begin{array}{l}\text { IG: } 20.4 \pm 4.6 \\
C G: 20.5 \pm 5.4\end{array}$ & $\begin{array}{l}\text { Balance board training } \\
\text { (home-based) }\end{array}$ & $\begin{array}{l}\text { Every day for } 30 \text { days then } 3 x \\
\text { week for the rest of the season } \\
(10-15 \mathrm{~min})\end{array}$ & $\begin{array}{l}\text { Total: } 140 \\
\text { IG: } 62 \\
\text { CG: } 78\end{array}$ & High \\
\hline Soligard et $\left.a\right|^{57}$ & Norway & $\begin{array}{l}\text { Under-15 and under-16 } \\
\text { teams }\end{array}$ & $15.4 \pm 0.7$ & $\begin{array}{l}\text { Neuromuscular training } \\
\text { (FIFA 11+) }\end{array}$ & $\begin{array}{l}\text { Every practice }(20 \mathrm{~min}) \text { and } \\
\text { game }(10 \mathrm{~min}) \\
2-5 \times \text { week depending on team }\end{array}$ & $\begin{array}{l}\text { Total: } 1892 \\
\text { IG: } 1055 \\
\text { CG: } 837\end{array}$ & High \\
\hline Steffen et $a l^{58}$ & Norway & Under-17 teams & $15.4 \pm 0.8$ & $\begin{array}{l}\text { Neuromuscular training } \\
\text { (FIFA 11) }\end{array}$ & $\begin{array}{l}\text { Every training for } 15 \text { sessions } \\
\text { then } 1 \times \text { week for the rest of } \\
\text { the season }(20 \mathrm{~min})\end{array}$ & $\begin{array}{l}\text { Total: } 2020 \\
\text { IG: } 1073 \\
\text { CG: } 947\end{array}$ & High \\
\hline Waldén et $a l^{59}$ & Sweden & Under-14 to 18 teams & $\begin{array}{l}I G: 14.0 \pm 1.2 \\
C G: 14.1 \pm 1.2\end{array}$ & $\begin{array}{l}\text { Neuromuscular training } \\
\text { (Knäkontroll) }\end{array}$ & $2 \times$ week ( $15 \mathrm{~min})$ & $\begin{array}{l}\text { Total: } 4564 \\
\text { IG: } 2479 \\
\text { CG: } 2085\end{array}$ & High \\
\hline Zebis et $a^{60}$ & Denmark & Under-18 teams & $15-18$ & $\begin{array}{l}\text { Using a lighter, smaller } \\
\text { football }\end{array}$ & $\begin{array}{l}\text { The different football was used } \\
\text { during matches and training }\end{array}$ & $\begin{array}{l}\text { Total: } 332 \\
\text { IG: } 147 \\
\text { CG: } 185\end{array}$ & High \\
\hline
\end{tabular}

${ }^{*}$ All interventions were for one season/year.

tSample size only relates to female football players.

¥The age of these participants includes women from other sports (volleyball and basketball).

Risk of bias, Cochrane Collaboration Risk of Bias Tool; CG, control group; CORE, exercises focused on the trunk and lower extremity; IG, intervention group; NCAA, National Collegiate Athletic Association; PEP, Prevent injury and Enhance Performance.

19.88)..$^{52}$ Four studies included female athletes from sports other than football ${ }^{4954}$ or included male and female football players together. ${ }^{5055}$ The remaining two studies were in national divisions and therefore included older participants. ${ }^{5156}$ Intervention adherence data as well as the education and resources provided to the teams are reported for each study in online supplementary appendix table 4 .

\section{Intervention components}

Neuromuscular exercise-based training (with multiple training components) was the most common intervention used in 9/12 studies. $^{49} 50$ 52-55 57-59 Two other studies in the review included only one exercise-based training component (one was a homebased balance training programme, ${ }^{56}$ and one an eccentric hamstring strengthening programme). ${ }^{51}$ The final study included in the review examined the effect of a lighter, smaller football on injury rate. ${ }^{60}$ As this was the only study with an intervention that was not exercise-based, it was not included in the meta-analyses. This study found a non-significant $22 \%$ greater risk of injury in the control group using the standard ball size compared with the intervention group who used the lighter and smaller ball. ${ }^{60}$

Regarding intervention components, the most common exercise-based training component was strength (10/11 studies), while agility and mobility exercises were used the least $(5 / 11$ studies; table 2). With respect to meeting training prescription guidelines, only 30\% (3/10) met the guidelines for strength, and $67 \%(6 / 9)$ met the guidelines for plyometric/power training.

\section{Effect of intervention on injury}

Table 3 presents the reported exposure hours and number of injuries for all studies (study by Zebis et $a l^{60}$ not included in the meta-analyses as it was not exercise-based). This includes overall reported injuries (primary aim) as well as specific lower-limb regions for ACL, knee, ankle, hip/groin and hamstring injuries (secondary aim). The GRADE rating of the evidence is presented in online supplementary appendix table 5 for each meta-analysis of exercise-based injury prevention programmes.

For overall injury (figure 2), we found low-level evidence (online supplementary appendix table 5) that there was a significant overall reduction of $22 \%$ in the intervention groups compared with the control groups for exercise-based programmes (total of 11773 participants; IRR $0.78,95 \%$ CI 0.64 to 0.95 ). For studies that included multiple training components, a significant reduction of $27 \%$ was observed (IRR 0.73 , $95 \%$ CI 0.59 to 0.91), while also being rated as low.

For ACL injuries (figure 3), there was a significant reduction of $45 \%$ (IRR $0.55,95 \%$ CI 0.32 to 0.92 ) when examining multiple training component studies. However, when the one study that examined only balance training by Söderman et $a l^{56}$ was included, this reduction was not significant at 38\% (IRR 
Table 2 Intervention components for exercise-based injury prevention programmes

\begin{tabular}{|c|c|c|c|c|c|c|c|}
\hline Study & Intervention & Agility & Balance & Mobility & Plyometric* & Running & Strength* \\
\hline Barber Foss et a $\left.\right|^{49}$ & $\begin{array}{l}\text { Neuromuscular training } \\
\text { (CORE intervention) }\end{array}$ & 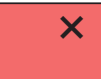 & $\checkmark$ & $x$ & $s^{\prime}$ & $x$ & $\sqrt{x}$ \\
\hline Emery and Meeuwisse $\mathrm{e}^{50}$ & $\begin{array}{l}\text { Neuromuscular training } \\
\text { (plus home-based balance training) }\end{array}$ & $x$ & $\checkmark$ & $\checkmark$ & $\sqrt{x}$ & $\checkmark$ & $\sqrt{x}$ \\
\hline Espinosa et $\left.a\right|^{51}$ & Eccentric hamstring exercises & $x$ & $\mathrm{x}$ & $\mathrm{X}$ & $x$ & $\mathrm{x}$ & $\sqrt{x}$ \\
\hline Gilchrist et $a P^{52}$ & $\begin{array}{l}\text { Neuromuscular training } \\
\text { (PEP programme) }\end{array}$ & $\checkmark$ & $x$ & $\checkmark$ & $v_{x}$ & $\checkmark$ & $\widehat{\checkmark}_{x}$ \\
\hline Heidt et $a l^{53}$ & $\begin{array}{l}\text { Neuromuscular training (Frappier Acceleration } \\
\text { Training programme) }\end{array}$ & $\checkmark$ & $\mathrm{x}$ & $\checkmark$ & $s^{\prime}$ & $\checkmark$ & $\sqrt{x}$ \\
\hline LaBella et $\left.a\right|^{54}$ & Neuromuscular training & $\checkmark$ & $\times$ & $\checkmark$ & $s_{1}$ & $\checkmark$ & $v_{x}$ \\
\hline Rössler et $a l^{55}$ & Neuromuscular training (FIFA 11+Kids) & $x$ & $\checkmark$ & $x$ & $s^{2}$ & $\checkmark$ & $s^{4}$ \\
\hline Söderman et $a P^{56}$ & $\begin{array}{l}\text { Balance board training } \\
\text { (home-based) }\end{array}$ & $x$ & $\checkmark$ & $x$ & $x$ & $x$ & $x$ \\
\hline Soligard et $a^{57}$ & $\begin{array}{l}\text { Neuromuscular training } \\
\text { (FIFA 11+) }\end{array}$ & $\checkmark$ & $\checkmark$ & $\checkmark$ & $s_{s}$ & $\checkmark$ & $\checkmark_{v}$ \\
\hline Steffen et $\left.a\right|^{58}$ & $\begin{array}{l}\text { Neuromuscular training } \\
\text { (FIFA 11) }\end{array}$ & $\checkmark$ & $\checkmark$ & $\mathrm{x}$ & $\sqrt{x}$ & $\checkmark$ & $\sqrt{x}$ \\
\hline Waldén et $a P^{59}$ & Neuromuscular training (Knäkontroll) & $x$ & $\checkmark$ & $x$ & $\checkmark^{\prime}$ & $\checkmark$ & $d^{\prime}$ \\
\hline Total (\%) & & $45 \%$ & $64 \%$ & $45 \%$ & $82 \%$ & $73 \%$ & $91 \%$ \\
\hline
\end{tabular}

$\checkmark$, included; $\mathbf{X}$, did not include; ${ }^{*}$, articles that included strength and plyometric components were assessed to determine if they met training prescription guidelines for strength and power respectively ${ }^{39}$ - small $\checkmark$ symbols in a green cell indicate the article met the muscular strength (included at least 2 sets, 8-15 repetitions or 20-30s duration, AND one progression (ie, increased intensity/difficulty)) or power (included at least 1 set, 3-15 repetitions or 10-30s duration, AND one progression (ie, increased intensity/difficulty)) guidelines, while small $\mathbf{X}$ symbols in an orange cell indicate they did not.

CORE, exercises focused on the trunk and lower extremity; PEP, Prevent injury and Enhance Performance.

$0.62,95 \%$ CI 0.37 to 1.05$)$. The level of evidence for the ACL meta-analyses was rated as low (online supplementary appendix table 5).

The reduction in knee injuries for the intervention groups compared with the control groups was not significant (figure 3), with the level of evidence for the knee injury meta-analyses rated as low (online supplementary appendix table 5). The overall reduction was $15 \%$ with all included studies (IRR $0.85,95 \% \mathrm{CI}$ 0.67 to 1.09 ), and $17 \%$ with only multiple training component studies (IRR 0.83 , 95\% CI 0.65 to 1.06 ).

The reduction in ankle injuries for the intervention groups compared with the control groups was not significant (figure 3),

Table 3 Exposure and injury data for all injury prevention programmes

\begin{tabular}{|c|c|c|c|c|c|c|c|c|c|}
\hline Study & Intervention & $\begin{array}{l}\text { Participants } \\
\text { analysed }\end{array}$ & Exposure hours & $\begin{array}{l}\text { Overall } \\
\text { injuries }\end{array}$ & $\mathrm{ACL}$ injuries & Knee injuries & $\begin{array}{l}\text { Ankle } \\
\text { injuries }\end{array}$ & $\begin{array}{l}\text { Hip/Groin } \\
\text { injuries }\end{array}$ & $\begin{array}{l}\text { Hamstring } \\
\text { injuries }\end{array}$ \\
\hline Barber Foss et al ${ }^{49}$ & $\begin{array}{l}\text { Neuromuscular training } \\
\text { (CORE intervention) }\end{array}$ & $\begin{array}{l}\text { IG: } 74 \\
\text { CG: } 68\end{array}$ & $\begin{array}{l}\text { IG: } 12120^{*} \\
\text { CG: } 10818^{*}\end{array}$ & $\begin{array}{l}\text { IG: } 25 \\
\text { CG: } 31\end{array}$ & $\begin{array}{l}\text { IG: } 0 \\
\text { CG: } 0\end{array}$ & $\begin{array}{l}\text { IG: } 11 \\
\text { CG: } 15\end{array}$ & $\begin{array}{l}\text { IG: } 6 \\
\text { CG: } 9\end{array}$ & NR & NR \\
\hline Emery and Meeuwisse ${ }^{50}$ & $\begin{array}{l}\text { Neuromuscular training } \\
\text { (plus home-based balance training) }\end{array}$ & $\begin{array}{l}\text { IG: } 161 \\
\text { CG: } 251\end{array}$ & $\begin{array}{l}\text { IG: } 10364 \\
\text { CG: } 16289\end{array}$ & $\begin{array}{l}\text { IG: } 27 \\
\text { CG: } 61\end{array}$ & NR & $\begin{array}{l}\text { IG: } 3 \\
\text { CG: } 6\end{array}$ & $\begin{array}{l}\text { IG: } 8 \\
\text { CG: } 23\end{array}$ & NR & NR \\
\hline Espinosa et $a l^{51}$ & Eccentric hamstring exercises & $\begin{array}{l}\text { IG: } 22 \\
\text { CG: } 21\end{array}$ & $\begin{array}{l}\text { IG: } 4827 \dagger \\
\text { CG: } 4608 t\end{array}$ & $\begin{array}{l}\text { IG: } 28 \\
\text { CG: } 23\end{array}$ & NR & NR & NR & NR & $\begin{array}{l}\text { IG: } 1 \\
\text { CG: } 5\end{array}$ \\
\hline Gilchrist et al ${ }^{52}$ & $\begin{array}{l}\text { Neuromuscular training } \\
\text { (PEP programme) }\end{array}$ & $\begin{array}{l}\text { IG: } 583 \\
\text { CG: } 852\end{array}$ & $\begin{array}{l}\text { IG: } 70440^{*} \\
\text { CG: } 105838^{*}\end{array}$ & NR & $\begin{array}{l}\text { IG: } 7 \\
\text { CG: } 18\end{array}$ & $\begin{array}{l}\text { IG: } 40 \\
\text { CG: } 58\end{array}$ & NR & NR & NR \\
\hline Heidt et $a l^{53}$ & $\begin{array}{l}\text { Neuromuscular training (Frappier } \\
\text { Acceleration Training programme) }\end{array}$ & $\begin{array}{l}\text { IG: } 42 \\
\text { CG: } 258\end{array}$ & $\begin{array}{l}\text { IG: } 9828 \ddagger \\
\text { CG: } 60372 \ddagger\end{array}$ & $\begin{array}{l}\text { IG: } 7 \\
\text { CG: } 91\end{array}$ & $\begin{array}{l}\text { IG: } 1 \\
\text { CG: } 8\end{array}$ & $\begin{array}{l}\text { IG: } 3 \\
\text { CG: } 29\end{array}$ & $\begin{array}{l}\text { IG: } 2 \\
\text { CG: } 26\end{array}$ & $\begin{array}{l}\text { IG: } 0 \\
\text { CG: } 3\end{array}$ & $\begin{array}{l}\text { IG: } 0 \\
\text { CG: } 3\end{array}$ \\
\hline LaBella et $\left.a\right|^{54}$ & Neuromuscular training & $\begin{array}{l}\text { IG: } 321 \\
\text { CG: } 333\end{array}$ & $\begin{array}{l}\text { IG: } 18278^{*} \\
\text { CG: } 15292^{*}\end{array}$ & $\begin{array}{l}\text { IG: } 32 \\
\text { CG: } 66\end{array}$ & $\begin{array}{l}\text { IG: } 0 \\
\text { CG: } 1\end{array}$ & $\begin{array}{l}\text { IG: } 1 \\
\text { CG: } 5\end{array}$ & $\begin{array}{l}\text { IG: } 6 \\
\text { CG: } 14\end{array}$ & NR & NR \\
\hline Rössler et $a^{55}$ & $\begin{array}{l}\text { Neuromuscular training (FIFA } \\
11+\text { Kids) }\end{array}$ & $\begin{array}{l}\text { IG: } 103 \\
\text { CG: } 68\end{array}$ & $\begin{array}{l}\text { IG: } 3931 \S \\
\text { CG: } 2932 \S\end{array}$ & $\begin{array}{l}\text { IG: } 5 \\
\text { CG: } 7\end{array}$ & $\begin{array}{l}\text { IG: } 0 \\
\text { CG: } 0\end{array}$ & $\begin{array}{l}\text { IG: } 2 \\
\text { CG: } 1\end{array}$ & $\begin{array}{l}\text { IG: } 0 \\
\text { CG: } 1\end{array}$ & $\begin{array}{l}\text { IG: } 1 \\
\text { CG: } 0\end{array}$ & NR \\
\hline Söderman et $a l^{56}$ & $\begin{array}{l}\text { Balance board training } \\
\text { (home-based) }\end{array}$ & $\begin{array}{l}\text { IG: } 62 \\
\text { CG: } 78\end{array}$ & $\begin{array}{l}\text { IG: } 8246 \S \\
\text { CG: } 9262 \S\end{array}$ & $\begin{array}{l}\text { IG: } 28 \\
\text { CG: } 31\end{array}$ & $\begin{array}{l}\text { IG: } 4 \\
\text { CG: } 1\end{array}$ & $\begin{array}{l}\text { IG: } 8 \\
\text { CG: } 6\end{array}$ & $\begin{array}{l}\text { IG: } 13 \\
\text { CG: } 14\end{array}$ & $\begin{array}{l}\text { IG: } 1 \\
\text { CG: } 0\end{array}$ & $\begin{array}{l}\text { IG: } 1 \\
\text { CG: } 7\end{array}$ \\
\hline Soligard et $a P^{57}$ & $\begin{array}{l}\text { Neuromuscular training } \\
\text { (FIFA 11+) }\end{array}$ & $\begin{array}{l}\text { IG: } 1055 \\
\text { CG: } 837\end{array}$ & $\begin{array}{l}\text { IG: } 49899 \\
\text { CG: } 45428\end{array}$ & $\begin{array}{l}\text { IG: } 161 \\
\text { CG: } 215\end{array}$ & NR & $\begin{array}{l}\text { IG: } 35 \\
\text { CG: } 58\end{array}$ & $\begin{array}{l}\text { IG: } 51 \\
\text { CG: } 52\end{array}$ & $\begin{array}{l}\text { IG: } 10 \\
\text { CG: } 9\end{array}$ & $\begin{array}{l}\text { IG: } 5 \\
\text { CG: } 8\end{array}$ \\
\hline Steffen et $\left.a\right|^{58}$ & $\begin{array}{l}\text { Neuromuscular training } \\
\text { (FIFA 11) }\end{array}$ & $\begin{array}{l}\text { IG: } 1073 \\
\text { CG: } 947\end{array}$ & $\begin{array}{l}\text { IG: } 66423 \\
\text { CG: } 65725\end{array}$ & $\begin{array}{l}\text { IG: } 242 \\
\text { CG: } 241\end{array}$ & $\begin{array}{l}\text { IG: } 4 \\
\text { CG: } 5\end{array}$ & $\begin{array}{l}\text { IG: } 37 \\
\text { CG: } 30\end{array}$ & $\begin{array}{l}\text { IG: } 79 \\
\text { CG: } 74\end{array}$ & $\begin{array}{l}\text { IG: } 6 \\
\text { CG: } 14\end{array}$ & NR \\
\hline Waldén et a $/^{59}$ & $\begin{array}{l}\text { Neuromuscular training } \\
\text { (Knäkontroll) }\end{array}$ & $\begin{array}{l}\text { IG: } 2479 \\
\text { CG: } 2085\end{array}$ & $\begin{array}{l}\text { IG: } 149214 \\
\text { CG: } 129084\end{array}$ & NR & $\begin{array}{l}\text { IG: } 7 \\
\text { CG: } 14\end{array}$ & $\begin{array}{l}\text { IG: } 49 \\
\text { CG: } 47\end{array}$ & NR & NR & NR \\
\hline Zebis et $a l^{60}$ & Using a lighter, smaller football & $\begin{array}{l}\text { IG: } 147 \\
\text { CG: } 185\end{array}$ & $\begin{array}{l}\text { IG: } 1119.3 \\
\text { CG: } 1559.1\end{array}$ & $\begin{array}{l}\text { IG: } 17 \\
\text { CG: } 29\end{array}$ & NR & $\begin{array}{l}\text { IG: } 2 \\
\text { CG: } 9\end{array}$ & $\begin{array}{l}\text { IG: } 7 \\
\text { CG: } 8\end{array}$ & $\begin{array}{l}\text { IG: } 0 \\
\text { CG: } 1\end{array}$ & $\begin{array}{l}\text { IG: } 1 \\
\text { CG: } 4\end{array}$ \\
\hline
\end{tabular}

Overall injuries for each study may have included just lower-limb or whole body injuries. Knee injuries incorporated all reported knee injuries that most likely included ACL injuries too.

${ }^{*}$ Calculated from athletic exposures (1 athletic exposure $=2$ exposure hours).

tData calculated based on the total exposure hours reported for all participants that was provided by the authors.

¥Data obtained by contacting the authors.

$\S$ Estimated exposure hours based on the assumption of 6 hours exposure per week per participant (two training sessions plus one game) for 39 weeks ${ }^{53}$ and then multiplied by the number of participants.

CG, control group; CORE, exercises focused on the trunk and lower extremity; IG, intervention group; NR, not reported; PEP, Prevent injury and Enhance Performance. 


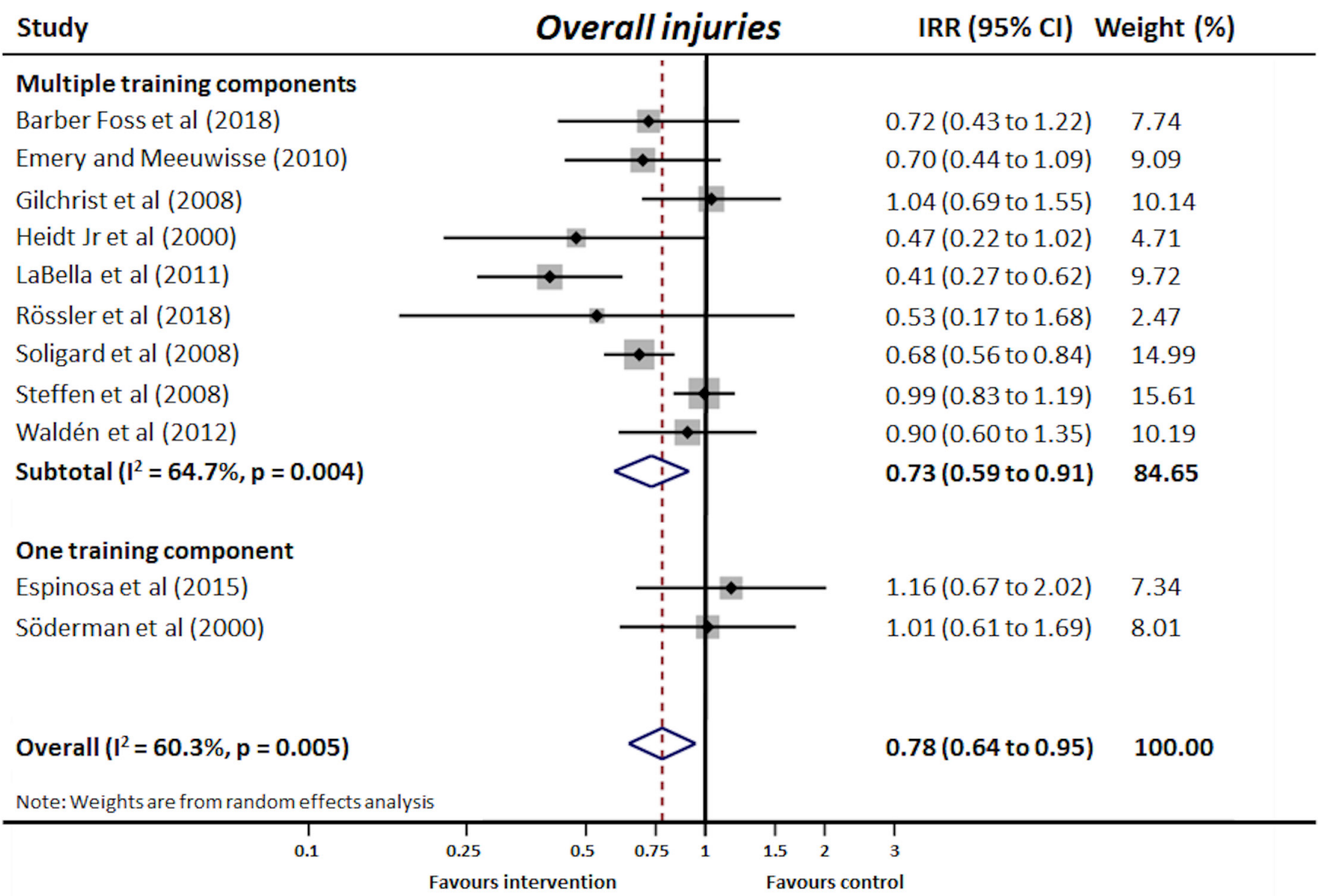

Figure 2 Meta-analysis examining the effectiveness of reducing overall injury for the exercise-based intervention groups compared with control groups. Note: the size of the boxes around each diamond are proportional to the weight of each study, and the horizontal lines represent the $95 \% \mathrm{Cl}$. The open diamond represents the pooled overall injury incidence rate ratio (IRR) and its width represents its $95 \%$ Cl. The studies by Espinosa et al ${ }^{51}$ and Söderman et $a l^{56}$ were separated due to only including one exercise-based training component. Studies by Gilchrist et a $P^{52}$ and Waldén et a $I^{59}$ only reported knee injuries, and thus the values for overall injuries are taken from knee injuries.

with the level of evidence for these meta-analyses rated as low (online supplementary appendix table 5). The overall reduction was $17 \%$ when all studies were included (IRR 0.83 , 95\% CI 0.65 to 1.07 ), and $22 \%$ with only multiple training component studies (IRR $0.78,95 \%$ CI 0.58 to 1.05 ).

There was low-level evidence (online supplementary appendix table 5) for a non-significant reduction in hip/groin injuries (figure 3). The overall reduction was $25 \%$ when all studies were included (IRR 0.75 , 95\% CI 0.41 to 1.40 ), and $29 \%$ with only multiple training component studies (IRR $0.71,95 \%$ CI 0.38 to 1.33).

Hamstring injuries had a significant reduction when all studies were included (figure 3) with a low level of evidence rating (online supplementary appendix table 5). There was a nonsignificant reduction when examining the two multiple training component studies (also rated as low; IRR 0.60, 95\% CI 0.21 to 1.71 ). The reduction for hamstring injuries when all studies were included was significant at 60\% (IRR 0.40, 95\% CI 0.17 to 0.95$)$.

Relationship between training components and injury reduction

The results of the meta-regression plots are shown in online supplementary appendix figure 6 . There were no significant associations $(p>0.05)$ found between the number of exercise-based training components included in the intervention and the IRR for all injuries (hamstring injuries were not examined due to the low number of studies reporting hamstring injuries). There appeared to be a negative relationship (eg, more training components reduced injury risk) for overall and knee injuries, however these relationships were non-significant $(p=0.123$ and 0.116$)$. This was supported by a reduction in $\tau^{2}$ for the overall and knee injury analyses. For ACL injury, the study by Söderman et al $l^{56}$ that only examined balance training strongly influenced the meta regression results (online supplementary appendix table 6). Removing this outlier, there was no association between the number of training components and the IRR for ACL, ankle and hip/groin injuries $(\mathrm{p}=0.441-0.954)$.

Injury incidence in women's football

Injury incidence data were reported for the non-intervention groups of included studies (online supplementary appendix table 7). Sample size and exposure hours for these groups are reported in table 3. Many included studies did not separate training and match injuries, and hence the injury incidence data reported here are both training and match injuries combined. The incidence of overall injury in women's football is reported as 3.42 per 1000 exposure hours (95\% CI 3.19 to 3.67). The highest injury rate per lower-limb region was ankle injuries $(0.97$ per 1000 exposure hours, 95\% CI 0.85 to 1.11 ), followed by knee 


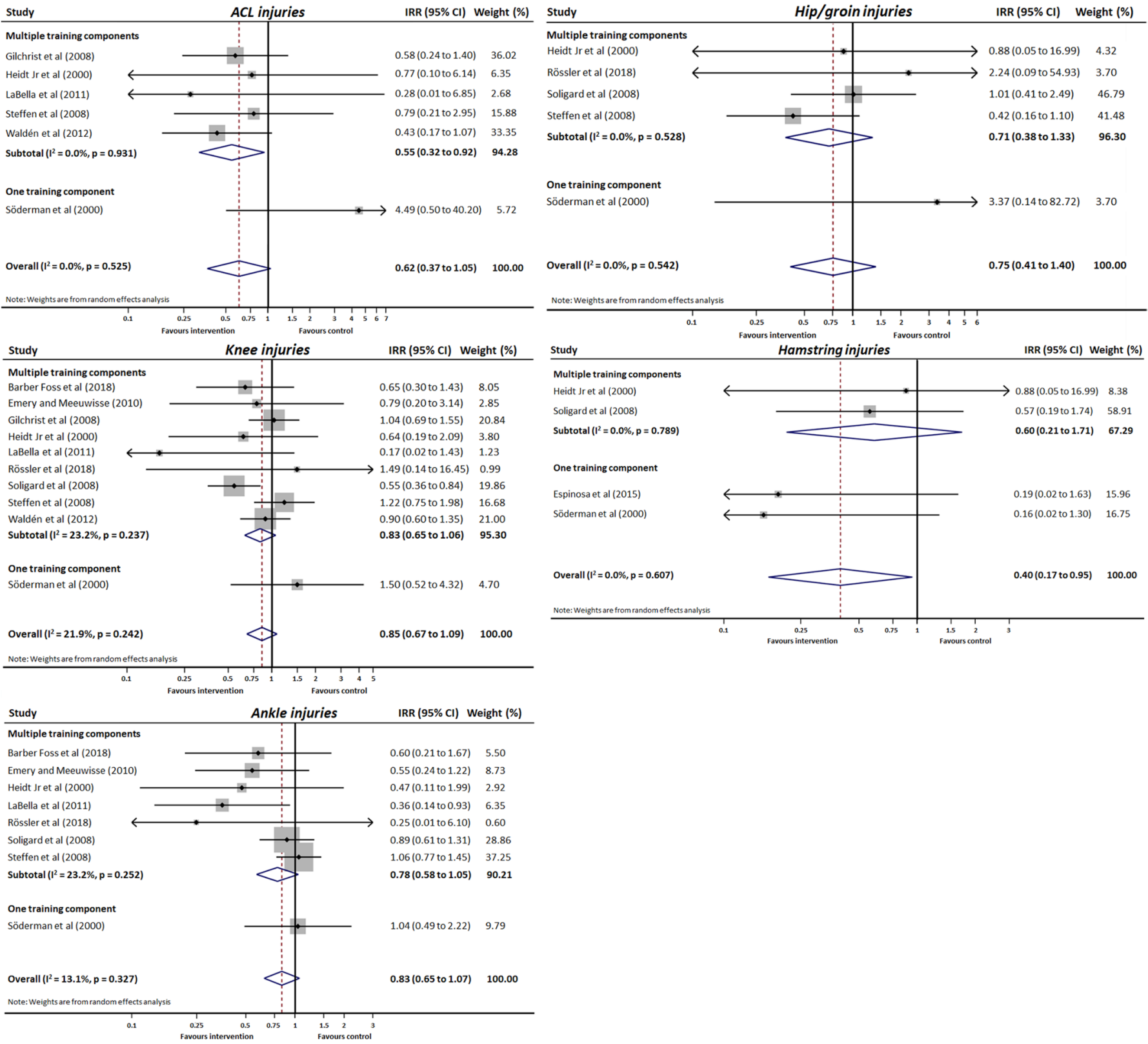

Figure 3 Meta-analysis examining the effectiveness of reducing $A C L$, knee, ankle, hip/groin and hamstring injuries for the exercise-based intervention groups compared with control groups. Note: the size of the boxes around each diamond are proportional to the weight of each study, and the horizontal lines represent the $95 \% \mathrm{Cl}$. The open diamond represents the pooled overall injury incidence rate ratio (IRR) and its width represents its $95 \% \mathrm{CI}$. For ACL injuries, the studies by Barber Foss et $a f^{49}$ and Rössler et $a f^{55}$ were unable to be included in the meta-analysis as both the intervention and control groups reported no ACL injuries. ${ }^{104}$ For all meta-analyses, studies reporting no injuries in one group (either intervention or control) had a fixed correction applied to both groups of 0.5 as suggested by the Cochrane Handbook for Systematic Reviews of Interventions. ${ }^{104}$

$(0.57,95 \%$ CI 0.51 to 0.64$)$, hamstring $(0.22,95 \%$ CI 0.15 to $0.32)$, hip/groin $(0.15,95 \%$ CI 0.10 to 0.21$)$ and ACL injuries $(0.12,95 \%$ CI 0.09 to 0.16$)$. Injury incidence rates were mostly unchanged when just examining adolescent teams ( $<18$ years), except for hamstring injuries with reduced incidence to 0.14 per 1000 exposure hours (95\% CI 0.08 to 0.23 ).

\section{DISCUSSION}

For female football (soccer) players, injury prevention programmes incorporating multiple exercise-based components can reduce overall injury rates $(27 \%)$ and ACL injury rates (45\%). Reduction rates were not as successful (22\% and 38\%, respectively) when including single-component programmes.
Nine out of the 11 exercise-based interventions included more than one training component, ${ }^{49} 5052-5557-59$ while one study included eccentric hamstring strengthening only, ${ }^{51}$ and another balance training only. ${ }^{56}$ When examining both single-component and multicomponent injury prevention programmes, exercisebased programmes can reduce hamstring injuries by $60 \%$. Greater reductions in overall and knee injuries were observed with a larger number of training components included in the intervention, but further studies would be required to increase the precision of these results. The incidence of overall injuries in women's football was 3.42 per 1000 exposure hours, with ankle injuries most common. 
Effectiveness of injury prevention programmes on overall injuries in women's football

Overall injuries among female football players $(n=11773)$ were reduced (22\%) with exercise-based injury prevention programmes (for single-component and multicomponent) and reduced by $27 \%$ when only examining multicomponent programmes. This $22 \%-27 \%$ reduction is lower than previously reported overall injury reduction rates of $27 \%-40 \%$ among male football players (using the FIFA 11/FIFA $11+$ ). ${ }^{61}$ The lower injury prevention effectiveness in female compared with male football players may reflect different responses to exercise-based training programmes in women and men. ${ }^{62}$ Additionally, the lower injury reduction rate in our current review may relate to the heterogeneity between injury prevention programmes (compared with the previous review of the FIFA 11 /FIFA $11+),{ }^{61}$ with some targeting particular injuries (eg, hamstring or ACL injuries) and some designed for other sports (eg, basketball) that may not be specific to the female football player. Designing an injury prevention programme specific to the athlete and the sport may facilitate greater buy-in and adherence from coaches and players, and increase the programme's effectiveness. ${ }^{63-65}$ Adherence is not known to differ between men and women, nor between football players and other female athletes, and future research should evaluate sex differences in response to different types of training, adherence and strategies to improve implementation and exercise fidelity in a female football context. ${ }^{66}$

\section{Effectiveness of injury prevention programmes on $\mathrm{ACL}$ injuries in women's football}

Multicomponent, exercise-based injury prevention programmes led to a $45 \%$ reduction in ACL injuries (IRR 0.55, 95\% CI 0.32 to 0.92 ), highlighting the effectiveness of such programmes in women's football. These results are consistent with ACL injury reduction rates of $41 \%-59 \%$ in all athletes (men and women), and the 39\%-60\% reduction reported for ACL injuries across various female sporting populations. ${ }^{35} 3867-70$ Despite the effectiveness of exercise-based ACL prevention programmes in women's football, they have less impact if they are not used, implemented correctly or sustained over time. ${ }^{71-73}$ The 'realworld' effect of injury prevention programmes may be reduced to as low as $13 \% .{ }^{74}$ Future studies should include planning and strategies to address known competency, organisational and leadership barriers to implementation, ${ }^{75}$ and improve monitoring and reporting methods. ${ }^{66}$

Effectiveness of injury prevention programmes on knee, ankle and hip/groin injuries in women's football

Knee, ankle and hip/groin injuries were reduced in female football players (knee 15\%-17\%; ankle 17\%-22\%; hip/groin 25\%-29\%), but additional studies are needed to improve the precision of these results. These injury reduction rates were lower than those reported previously in male soccer players (knee 32\%-58\%; ankle $30 \%-60 \%$; hip/groin 47\%), ${ }^{76-78}$ but the reductions of $15 \%, 17 \%$ and $25 \%$ for knee, ankle and hip/groin injuries in multicomponent programmes respectively, may be meaningful in the context of sport, where any additional increase in player availability (particularly to higher ranked players) is important for team success. ${ }^{79} 80$

The current findings should be interpreted with caution considering the low level of evidence, and knowing that the definitions of injuries, particularly of the hip/groin varied considerably-three reported 'groin' injuries, ${ }^{55} 58$ one specifically reported 'hip/ groin', ${ }^{57}$ and data were extracted for hip flexor and groin strains in one study. ${ }^{53}$ As with overall injuries, it is difficult to compare our smaller reduction rates with previous studies due to the many confounding factors (eg, injury definitions, intervention length, adherence) other than sex.

\section{Effectiveness of injury prevention programmes on hamstring} injuries in women's football

Hamstring injuries were reduced by 40\%-60\% from all studies, comparable to rates in male collegiate $(63 \%),{ }^{77}$ and amateur $(48 \%)^{81}$ soccer players using the FIFA $11+$. The single-component injury prevention programmes, including progressive eccentric hamstring strengthening exercises (81\% reduction), ${ }^{51}$ or balance training (84\% reduction), ${ }^{56}$ were more effective than multiple training component programmes. ${ }^{53} 57$ The high hamstring injury reduction rates with eccentric exercise ${ }^{51}$ would be expected, given the significant reductions (45\%-77\%) observed when an eccentric hamstring exercise is adhered to. ${ }^{65}$ Since postexercise muscle soreness ${ }^{82} 83$ might affect adherence, rescheduling the eccentric exercise component to after training ${ }^{84}$ may assist with adherence and hamstring injury reduction. Interestingly, the balance-only training resulted in large significant reduction in hamstring injuries. $^{56}$ While multicomponent prevention programmes include hamstring strengthening exercises, only two multi-component studies ${ }^{53}$ reported hamstring injury rates, and further investigation is required.

\section{Influence of included training components and clinical implications}

The exercise-based injury prevention programmes (singlecomponent and multi-component) were most effective in reducing ACL injuries (38\% reduction), when compared with overall injuries, knee, ankle and hip/groin injuries (22\%, 15\%, 17\% and 25\% reduction, respectively). Given the high short-term and long-term burden of ACL injuries, ${ }^{202385}$ ACL prevention programmes often include neuromuscular exercises focusing on lower-limb alignment during activities specific to ACL injury mechanisms, aligning with current clinical practice guidelines. ${ }^{28} 298687$ While these activities may reduce all lower-limb musculoskeletal injuries, increased training specificity may be needed to further reduce knee, ankle and hip/groin injuries. For example, targeted progressive hip adductor strengthening to reduce groin injuries, ${ }^{88} 89$ load management strategies for chronic soft tissues or joint conditions ${ }^{90}$ or football skills to address sport-specific injury mechanisms and technical skills which may contribute to injury. ${ }^{28}$

The number of included training components was associated with greater injury risk reduction for overall and knee injuries, but these relationships were imprecise. This association was supported by a reduction in $\tau^{2}$ values, and consistent with the systematic review for ACL injuries in female athletes of various sports. ${ }^{38}$ Our findings may reflect low statistical power due to the small number of studies (especially for analysis of body regions), and/or the high variability between studies for each training component. Further research is needed to determine the optimal combination of training components in injury prevention programmes in women's football.

\section{Other injury prevention programmes}

Other than exercise-based strategies, this review identified only one RCT examining a different injury reduction strategy; a smaller and lighter football, with a non-significant injury reduction in adolescent soccer players. ${ }^{60}$ Other strategies such as training load, well-being monitoring, sleep or nutrition have yet to be investigated with the rigour of a full-scale RCT, and may warrant further 
investigation in women's football. The education and support provided to coaching staff and players in the included studies in this review is reported in online supplementary appendix table 4 . Improving coach self-efficacy through education and training for coaches of female teams should be a priority in women's football given the potential to improve adherence to injury prevention programmes. ${ }^{91}$ Furthermore, many coaches may lack sex-specific experience due to the rapid increase in participation and volunteer nature of women's sub-elite football.

\section{Incidence of injuries in women's football}

The overall (3.42 per 1000 exposure hours) and ACL, knee, ankle, hip/groin and hamstring injury incidence rates in women's football extend current data, which is mostly limited to ACL injury rates in senior elite men's football. ${ }^{1992} 93$ The average incidence of knee injury (0.57 per 1000 exposure hours) for predominantly female youth players in the current study is similar to previous reports in male senior elite-level (0.47-0.54 per 1000 hours), ${ }^{94}{ }^{95}$ and male collegiate-level (estimated at $0.47-0.59$ per 1000 hours) ${ }^{9697}$ soccer players. The incidence of ACL injury of 0.12 per 1000 exposure hours for predominately youth players in the current study is higher than senior elite-level $(0.06-0.11)^{92} 9495$ and collegiatelevel $(0.06-0.09)^{96} 9899$ female football players. Consistent with previous studies, ${ }^{100}$ the higher ACL injury rates in female than male youth players, reinforces the need for investment of resources towards effective injury risk reduction strategies into female youth and subelite programmes. Targeting developing players and player programmes is a priority, to ensure these players are provided the best opportunities to progress to senior football with optimal wellbeing and performance.

\section{Limitations}

The current study has inherent limitations due to the low-level of evidence, inconsistent definitions and reporting of injury and exposure hours. Additionally, our search strategy did not include any medical subject headings, which may have limited our search results. Large CIs indicate uncertainty in results in some analyses. This is largely due to studies not reporting all injury types and being excluded from meta-analyses, or those studies having a low injury incidence. ${ }^{53-56}$ Eleven studies were included for the overall injuries meta-analysis, however two studies ${ }^{5259}$ only assessed knee injuries, which were extrapolated in the current review to overall injuries. This process resulted in a conservative (but already significant) estimate of the injury reduction effect for overall injuries. A sensitivity analysis removing these two studies revealed the intervention effect to be greater for overall injury risk. The definition of overall injuries was also inconsistent or unclear and could refer to only lower-limb injuries or whole body (including upper body, head, neck), which could underestimate the IRR for overall injuries, if upper limb injuries were not recorded. This review did not examine upper limb injuries, with only three of the included studies reporting upper limb injuries separately. ${ }^{55} 5860$ Due to inconsistent reporting in the included studies, injuries and re-injuries were pooled together for this review as only a few studies separated re-injuries in their reporting. Intervention bias may be evident as many studies were designed with the primary aim to reduce ACL injuries. In addition, some athletes may have been exposed to additional interventions (exercise-based or education-based) outside of the study protocols, as part of their normal routine, but monitoring of such additional interventions was not recorded or feasible in many studies.

The meta-regressions examining the relationship between the number of included training components and injury risk reduction was limited, as it did not factor in many other influences (such as length of time of each component or adherence to each component) and contained a limited amount of studies. Further studies are needed to find the optimal dosage of exercise-based injury prevention programmes, with clear reporting on training dose (duration and frequency) and adherence. Our reporting of injury incidence does not reflect consensus statements that suggest reporting training and match injuries separately. ${ }^{101}$ However, we were unable to separate training and match injuries due to many included studies not reporting training and match injuries independent of each other. ${ }^{49-5154}$ Also, our a priori decision to convert one athletic exposure to two exposure hours (based on previous research $)^{37}$ may have resulted in an underestimation of our injury incidence data if the true session length of each exposure was $<2$ hours. Four included studies in the review reported athletic exposures (school/college teams in the USA), ${ }^{49} 52-54$ and using a shorter duration for each athletic exposure (60 or $90 \mathrm{~min}$ ) in these studies would have resulted in higher injury incidence results. Finally, while 11/12 studies were rated as a high risk of bias (table 1), the Cochrane Collaboration Risk of Bias Tool V.1 has inherent limitations to assess RCTs evaluating injury prevention programmes, given the inability for participants (players, coaches and programme deliverers) to be blinded (online supplementary appendix table 3). If this domain was removed, five studies would be upgraded to an unclear risk of bias.

\section{Future directions}

Future research should explore the sex-specific reasons for the smaller injury reduction rates seen in this review, compared with those previously reported for men's soccer. Understanding aspects of the injury prevention programmes, such as the impact of sport specificity and the relative effectiveness of components, may enhance injury prevention efforts. The influence of sex-specific player and staff support, education, adherence and response to injury prevention programmes should be explored. There is also an urgent need for further research across all football codes (ie, no rugby, Gaelic, American or Australian football forms could be included) to understand the implications of different codes on injury risk, and on reduction strategies. ${ }^{15} 93$ Given the increasing professionalism of all women's football codes, and evolving athlete physical characteristics and injury profiles, injury surveillance should also extend to all injury types. Future injury prevention programmes should consider the context-specific playing group (ie, sex, age, level of competition, injury profile), staffing, training format and facilities ${ }^{28} 102103$ to inform content, and optimise adherence and implementation strategies. High-quality RCTs are also urgently needed, with improved methods of adherence monitoring, strategies to address female football-specific barriers and drivers of implementation, following implementation science and behaviour change theories. ${ }^{6675}$

\section{CONCLUSION}

Low-quality evidence suggests that multicomponent exercise-based injury prevention programmes reduced overall and ACL injuries by $27 \%$ and $45 \%$, respectively. Reductions of $17 \%, 22 \%$ and $29 \%$ were observed for knee, ankle and hip/groin injuries, but these imprecise findings reflect heterogeneity and/or lack of statistical power. Exercise-based strategies (both single-component and multicomponent) reduced hamstring injuries by $60 \%$. A larger number of included training components was associated with greater injury risk reduction for overall and knee injuries, but further studies are required to increase the precision of these findings. Given the high burden of overall and specifically ACL injuries, the challenge now is to implement these programmes into women's soccer, with best 
evidence suggesting multicomponent exercise-based injury prevention programmes can reduce injury risk.

\section{What is already known?}

Women's football is one of the fastest growing sports worldwide, at all levels.

- Injury risk differs between male and female athletes.

- Injury prevention programmes can reduce $A C L$ injuries in female athletes across a variety of sports (eg, handball, basketball).

- Injury prevention programmes can reduce overall injuries in men's football.

\section{What are the new findings?}

- In women's football (soccer), multicomponent exercise-based injury prevention programmes reduce overall injuries by $27 \%$ and $\mathrm{ACL}$ injuries by $45 \%$.

- There appears to be greater reductions in overall and knee injuries with a larger number of training components included in the intervention.

Twitter Kay M Crossley @kaymcrossley, Brooke E Patterson @Knee_Howells, Adam G Culvenor @agculvenor, Andrea M Bruder @AndreaBruder, Andrea B Mosler @AndreaBMosler and Benjamin F Mentiplay @MentiplayB

Contributors $\mathrm{KMC}$ conceived and designed the study. BFM, ABM, AMB, BEP and AGC conducted the search, quality appraisal and data extraction. All authors contributed to interpretation of data and analysis. BFM, KMC, BEP and AGC drafted the manuscript with input from ABM and AMB. All authors have read and approved the final manuscript.

Funding Support for this study was provided by an Australian Football League (AFL) Research Board Grant. BEP is supported by a National Health and Medical Research Council (NHMRC) postgraduate scholarship (no. 1114296). AGC is supported by an NHMRC Early Career Fellowship (no. 1121173). ABM is supported by an NHMRC Early Career Fellowship (No. 1156674).

Competing interests None declared.

Patient consent for publication Not required.

Provenance and peer review Not commissioned; externally peer reviewed.

Open access This is an open access article distributed in accordance with the Creative Commons Attribution Non Commercial (CC BY-NC 4.0) license, which permits others to distribute, remix, adapt, build upon this work non-commercially, and license their derivative works on different terms, provided the original work is properly cited, appropriate credit is given, any changes made indicated, and the use is non-commercial. See: http://creativecommons.org/licenses/by-nc/4.0/.

\section{ORCID iDs}

Kay M Crossley http://orcid.org/0000-0001-5892-129X

Brooke E Patterson http://orcid.org/0000-0002-6570-5429

Adam G Culvenor http://orcid.org/0000-0001-9491-0264

Andrea M Bruder http://orcid.org/0000-0001-5422-5756

Andrea B Mosler http://orcid.org/0000-0001-7353-2583

Benjamin F Mentiplay http://orcid.org/0000-0002-4360-8310

\section{REFERENCES}

1 AFL. Australian Football League (AFL) Annual Report 2017. Melbourne, 2018.

2 UEFA. Women's football across the national associations 2016/17 2017.

3 Martínez-Lagunas V, Niessen M, Hartmann U. Women's football: player characteristics and demands of the game. I Sport Health Sci 2014;3:258-72.

4 Eime RM, Young JA, Harvey JT, et al. A systematic review of the psychological and social benefits of participation in sport for adults: informing development of a conceptual model of health through sport. Int J Behav Nutr Phys Act 2013;10:135.

5 Eime RM, Young JA, Harvey JT, et al. A systematic review of the psychological and social benefits of participation in sport for children and adolescents: informing development of a conceptual model of health through sport. Int I Behav Nutr Phys Act 2013;10:98.

6 López-Valenciano A, Ruiz-Pérez I, Garcia-Gómez A, et al. Epidemiology of injuries in professional football: a systematic review and meta-analysis. Br I Sports Med 2019:bjsports-2018-099577.
7 Fortington LV, Finch CF. Priorities for injury prevention in women's Australian football: a compilation of national data from different sources. BMJ Open Sport Exerc Med 2016;2:e000101.

8 Smith HC, Vacek P, Johnson RJ, et al. Risk factors for anterior cruciate ligament injury: a review of the literature-part 2: hormonal, genetic, cognitive function, previous injury, and extrinsic risk factors. Sports Health 2012;4:155-61.

9 Toohey LA, Drew MK, Cook JL, et al. Is subsequent lower limb injury associated with previous injury? A systematic review and meta-analysis. Br I Sports Med 2017; 51:1670-8

10 Freckleton G, Pizzari T. Risk factors for hamstring muscle strain injury in sport: a systematic review and meta-analysis. Br J Sports Med 2013;47:351-8.

11 Ryan J, DeBurca N, Mc Creesh K. Risk factors for groin/hip injuries in field-based sports: a systematic review. Br J Sports Med 2014;48:1089-96.

12 Eime RM, Casey MM, Harvey JT, et al. Socioecological factors potentially associated with participation in physical activity and sport: a longitudinal study of adolescent girls. J Sci Med Sport 2015;18:684-90.

13 Montalvo AM, Schneider DK, Silva PL, et al. 'What's my risk of sustaining an ACL injury while playing football (soccer)?' a systematic review with meta-analysis. $\mathrm{Br}$ J Sports Med 2019:53:1333-40.

14 Dick RW. Is there a gender difference in concussion incidence and outcomes? $\mathrm{Br} J$ Sports Med 2009;43:i46-50

15 Prien A, Grafe A, Rössler R, et al. Epidemiology of head injuries focusing on concussions in team contact sports: a systematic review. Sports Med 2018:48:953-69.

16 Boling M, Padua D, Marshall S, et al. Gender differences in the incidence and prevalence of patellofemoral pain syndrome. Scand I Med Sci Sports 2010;20:725-30

17 Murphy DF, Connolly DA, Beynnon BD. Risk factors for lower extremity injury: a review of the literature. Br J Sports Med 2003;37:13-29.

18 Cross KM, Gurka KK, Saliba S, et al. Comparison of hamstring strain injury rates between male and female intercollegiate soccer athletes. Am I Sports Med 2013:41:742-8

19 Waldén $M$, Hägglund $M$, Ekstrand J. The epidemiology of groin injury in senior football: a systematic review of prospective studies. Br J Sports Med 2015;49:792-7.

20 Ardern CL, Taylor NF, Feller JA, et al. Fifty-five per cent return to competitive sport following anterior cruciate ligament reconstruction surgery: an updated systematic review and meta-analysis including aspects of physical functioning and contextual factors. Br I Sports Med 2014;48:1543-52.

21 Liptak MG, Angel KR. Return to play and player performance after anterior cruciate ligament injury in elite Australian rules football players. Orthop J Sports Med 2017:5:2

22 Culvenor AG, Collins NJ, Guermazi A, et al. Early knee osteoarthritis is evident one year following anterior cruciate ligament reconstruction: a magnetic resonance imaging evaluation. Arthritis Rheumatol 2015;67:946-55.

23 Filbay SR, Culvenor AG, Ackerman IN, et al. Quality of life in anterior cruciate ligament-deficient individuals: a systematic review and meta-analysis. $\mathrm{Br}$ I Sports Med 2015;49:1033-41.

24 Moses B, Orchard J, Orchard J. Systematic review: annual incidence of ACL injury and surgery in various populations. Res Sports Med 2012;20:157-79.

25 Meeuwisse WH, Tyreman H, Hagel B, et al. A dynamic model of etiology in sport injury: the recursive nature of risk and causation. Clinical Journal of Sport Med 2007:17:215-9.

26 Leppänen M, Aaltonen S, Parkkari J, et al. Interventions to prevent sports related injuries: a systematic review and meta-analysis of randomised controlled trials. Sports Med 2014:44:473-86.

27 Renstrom $\mathrm{P}$, Ljungqvist $\mathrm{A}$, Arendt $\mathrm{E}$, et al. Non-Contact ACL injuries in female athletes: an international Olympic Committee current concepts statement. $\mathrm{Br}$ J Sports Med 2008;42:394-412.

28 Bahr R, Krosshaug T. Understanding injury mechanisms: a key component of preventing injuries in sport. Br J Sports Med 2005;39:324-9.

29 Dai B, Mao D, Garrett WE, et al. Anterior cruciate ligament injuries in soccer: loading mechanisms, risk factors, and prevention programs. J Sport Health Sci 2014:3:299-306.

30 Impellizzeri FM, Bizzini M, Dvorak J, et al. Physiological and performance responses to the FIFA 11+ (Part 2): a randomised controlled trial on the training effects. J Sports Sci 2013:31:1491-502.

31 Bizzini M, Dvorak J. Fifa 11+: an effective programme to prevent football injuries in various player groups worldwide-a narrative review. $\mathrm{Br}$ I Sports Med 2015;49:577-9

32 Mandelbaum BR, Silvers $\mathrm{HJ}$, Watanabe DS, et al. Effectiveness of a neuromuscular and proprioceptive training program in preventing anterior cruciate ligament injuries in female athletes. Am J Sports Med 2005:33:1003-10.

33 Hägglund $M$, Waldén $M$, Atroshi I. Preventing knee injuries in adolescent female football players - design of a cluster randomized controlled trial [NCT00894595]. BMC Musculoskelet Disord 2009:10:75.

34 Donaldson A, Cook J, Gabbe B, et al. Bridging the gap between content and context: establishing expert consensus on the content of an exercise training program to prevent lower-limb injuries. Clin I Sport Med 2015;25:221-9. 
35 Webster KE, Hewett TE. Meta-Analysis of meta-analyses of anterior cruciate ligament injury reduction training programs. J Orthop Res 2018;36:2696-708.

36 Moher D, Liberati A, Tetzlaff J, et al. Preferred reporting items for systematic reviews and meta-analyses: the PRISMA statement. PLOS Med 2009;6:e1000097.

37 Michaelidis M, Koumantakis GA. Effects of knee injury primary prevention programs on anterior cruciate ligament injury rates in female athletes in different sports: a systematic review. Phys Ther Sport 2014;15:200-10.

38 Taylor JB, Waxman JP, Richter SJ, et al. Evaluation of the effectiveness of anterior cruciate ligament injury prevention programme training components: a systematic review and meta-analysis. Br J Sports Med 2015;49:79-87.

39 American College of Sports Medicine. Progression models in resistance training for healthy adults. Med Sci Sports Exerc 2009;41:687-708.

40 Behm DG, Faigenbaum AD, Falk B, et al. Canadian Society for exercise physiology position paper: resistance training in children and adolescents. Appl Physiol Nutr Metab 2008;33:547-61.

41 Higgins JPT, Altman DG, Gotzsche PC, et al. The Cochrane collaboration's tool for assessing risk of bias in randomised trials. BMJ 2011;343:d5928.

42 Landis JR, Koch GG. The measurement of observer agreement for categorical data. Biometrics 1977:33:159-74

43 Higgins JPTet al. Measuring inconsistency in meta-analyses. BMJ 2003;327:557-60

44 GRADE Working Group. Grading quality of evidence and strength of recommendations. BMJ 2004;328:1490.

45 Ryan R, Hill S. How to grade the quality of the evidence. Cochrane consumers and communication group. version 3.02016

46 Guyatt GH, Oxman AD, Kunz R, et al. Grade guidelines 6. rating the quality of evidence-imprecision. J Clin Epidemiol 2011;64:1283-93.

47 Bruder AM, Shields N, Dodd KJ, et al. Prescribed exercise programs may not be effective in reducing impairments and improving activity during upper limb fracture rehabilitation: a systematic review. J Physiother 2017;63:205-20.

48 Guyatt GH, Oxman AD, Montori V, et al. GRADE quidelines: 5. Rating the quality of evidence--publication bias. J Clin Epidemiol 2011;64:1277-82.

49 Barber Foss KD, Thomas S, Khoury JC, et al. A school-based neuromuscular training program and sport-related injury incidence: a prospective randomized controlled clinical trial. J Athl Train 2018;53:20-8.

50 Emery CA, Meeuwisse WH. The effectiveness of a neuromuscular prevention strategy to reduce injuries in youth soccer: a cluster-randomised controlled trial. $\mathrm{Br} J$ Sports Med 2010;44:555-62.

51 Espinosa GDA, Pöyhönen T, Aramendi JF, et al. Effects of an eccentric training programme on hamstring strain injuries in women football players. Biomed Hum Kinet 2015;7:125-34

52 Gilchrist J, Mandelbaum BR, Melancon $\mathrm{H}$, et al. A randomized controlled trial to prevent noncontact anterior cruciate ligament injury in female collegiate soccer players. Am J Sports Med 2008;36:1476-83.

53 Heidt RS, Sweeterman LM, Carlonas RL, et al. Avoidance of soccer injuries with preseason conditioning. Am J Sports Med 2000;28:659-62.

54 LaBella CR, Huxford MR, Grissom J, et al. Effect of neuromuscular warm-up on injuries in female soccer and Basketball athletes in urban public high schools. Arch Pediatr Adolesc Med 2011;165:1033-40.

55 Rössler $\mathrm{R}$, Junge $\mathrm{A}$, Bizzini $\mathrm{M}$, et al. A multinational cluster randomised controlled trial to assess the efficacy of '11+ Kids': a warm-up programme to prevent injuries in children's football. Sports Med 2018;48:1493-504.

56 Söderman K, Werner S, Pietilä T, et al. Balance board training: prevention of traumatic injuries of the lower extremities in female soccer players? A prospective randomized intervention study. Knee Surg Sports Traumatol Arthrosc 2000;8:356-63.

57 Soligard T, Myklebust G, Steffen K, et al. Comprehensive warm-up programme to prevent injuries in young female footballers: cluster randomised controlled trial. BMJ 2008;337:a2469

58 Steffen $\mathrm{K}$, Myklebust G, Olsen OE, et al. Preventing injuries in female youth football - a cluster-randomized controlled trial. Scand J Med Sci Sports 2008;18:605-14

59 Waldén M, Atroshi I, Magnusson H, et al. Prevention of acute knee injuries in adolescent female football players: cluster randomised controlled trial. BMJ 2012;344:e3042

60 Zebis MK, Thorborg K, Andersen LL, et al. Effects of a lighter, smaller football on acute match injuries in adolescent female football: a pilot cluster-randomized controlled trial. J Sports Med Phys Fitness 2018;58:644-50.

61 Al Attar WSA, Alshehri MA. A meta-analysis of meta-analyses of the effectiveness of FIFA injury prevention programs in soccer. Scand J Med Sci Sports 2019:29:1846-55

62 Shephard RJ. Exercise and training in women, part I: influence of gender on exercise and training responses. Can J App/ Physiol 2000;25:19-34.

63 Steffen K, Emery CA, Romiti M, et al. High adherence to a neuromuscular injury prevention programme (FIFA 11+) improves functional balance and reduces injury risk in Canadian youth female football players: a cluster randomised trial. $\mathrm{Br}$ J Sports Med 2013;47:794-802.

64 Soligard T, Nilstad A, Steffen K, et al. Compliance with a comprehensive warm-up programme to prevent injuries in youth football. Br J Sports Med 2010;44:787-93.
65 Goode AP, Reiman MP, Harris L, et al. Eccentric training for prevention of hamstring injuries may depend on intervention compliance: a systematic review and metaanalysis. Br J Sports Med 2015;49:349-56.

66 O'Brien J, Finch CF. A systematic review of core implementation components in team ball sport injury prevention trials. Injury Prevention 2014;20:357-62.

67 Hewett TE, Ford KR, Myer GD. Anterior cruciate ligament injuries in female athletes: Part 2, a meta-analysis of neuromuscular interventions aimed at injury prevention. Am J Sports Med 2006;34:490-8.

$68 \mathrm{Yoo} \mathrm{JH}$, Lim BO, Ha M, et al. A meta-analysis of the effect of neuromuscular training on the prevention of the anterior cruciate ligament injury in female athletes. Knee Surg Sports Traumatol Arthrosc 2010;18:824-30.

69 Sugimoto D, Myer GD, McKeon JM, et al. Evaluation of the effectiveness of neuromuscular training to reduce anterior cruciate ligament injury in female athletes: a critical review of relative risk reduction and numbers-needed-to-treat analyses. $\mathrm{Br} J$ Sports Med 2012;46:979-88.

70 Myer GD, Sugimoto D, Thomas S, et al. The influence of age on the effectiveness of neuromuscular training to reduce anterior cruciate ligament injury in female athletes. Am J Sports Med 2013;41:203-15.

71 Donaldson A, Lloyd DG, Gabbe BJ, et al. We have the programme, what next? planning the implementation of an injury prevention programme. Injury Prevention 2017:23:273-80

72 Slauterbeck JR, Choquette R, Tourville TW, et al. Implementation of the FIFA $11+$ injury prevention program by high school athletic teams did not reduce lower extremity injuries: a cluster randomized controlled trial. Am J Sports Med 2019;47:2844-52

73 Twomey D, Finch C, Roediger E, et al. Preventing lower limb injuries: is the latest evidence being translated into the football field? J Sci Med Sport 2009;12:452-6.

74 Åman M, Larsén K, Forssblad M, et al. A nationwide follow-up survey on the effectiveness of an implemented neuromuscular training program to reduce acute knee injuries in soccer players. Orthop J Sports Med 2018;6:2.

75 Donaldson A, Finch CF. Applying implementation science to sports injury prevention. Br J Sports Med 2013;47:473-5.

76 Thorborg K, Krommes KK, Esteve E, et al. Effect of specific exercise-based football injury prevention programmes on the overall injury rate in football: a systematic review and meta-analysis of the FIFA 11 and 11+ programmes. Br J Sports Med 2017:51:562-71.

77 Silvers-Granelli H, Mandelbaum B, Adeniji O, et al. Efficacy of the FIFA 11+ injury prevention program in the collegiate male soccer player. Am J Sports Med 2015;43:2628-37.

78 Owoeye OB, Akinbo SR, Tella BA, et al. Efficacy of the FIFA 11+ warm-up programme in male youth football: a cluster randomised controlled trial. J Sports Sci Med 2014;13:321-8.

79 Hoffman DT, Dwyer DB, Bowe SJ, et al. Is injury associated with team performance in elite Australian football? 20 years of player injury and team performance data that include measures of individual player value. Br J Sports Med 2019:bjsp orts-2018-100029.

80 Hägglund $M$, Waldén $M$, Magnusson $H$, et al. Injuries affect team performance negatively in professional football: an 11-year follow-up of the UEFA champions League injury study. Br J Sports Med 2013;47:738-42.

81 Nouni-Garcia R, Carratala-Munuera C, Orozco-Beltran D, et al. Clinical benefit of the FIFA 11 programme for the prevention of hamstring and lateral ankle ligament injuries among amateur soccer players. Injury Prevention 2018:24:149-54.

82 O'Brien J, Finch CF. The implementation of musculoskeletal injury-prevention exercise programmes in team ball sports: a systematic review employing the RE-AIM framework. Sports Med 2014;44:1305-18.

83 Lindblom H, Carlfjord S, Hägglund M. Adoption and use of an injury prevention exercise program in female football: a qualitative study among coaches. Scand J Med Sci Sports 2018;28:1295-303.

84 Whalan M, Lovell R, Steele JR, et al. Rescheduling Part 2 of the 11+ reduces injury burden and increases compliance in semi-professional football. Scand J Med Sci Sports 2019;29:1941-51.

85 Risberg MA, Oiestad BE, Gunderson R, et al. Changes in knee osteoarthritis, symptoms, and function after anterior cruciate ligament reconstruction: a 20-year prospective follow-up study. Am J Sports Med 2016:44:1215-24.

86 Carlson VR, Sheehan FT, Boden BP. Video analysis of anterior cruciate ligament (ACL) injuries: a systematic review. JBJS Rev 2016;4. doi:10.2106/JBJS.RVW.15.00116

87 Arundale AJH, Bizzini M, Giordano A, et al. Exercise-Based knee and anterior cruciate ligament injury prevention. J Orthop Sports Phys Ther 2018;48:A1-42.

88 Harøy J, Thorborg K, Serner A, et al. Including the Copenhagen adduction exercise in the FIFA $11+$ provides missing eccentric hip adduction strength effect in male soccer players: a randomized controlled trial. Am J Sports Med 2017;45:3052-9.

89 Harøy J, Clarsen B, Wiger EG, et al. The adductor strengthening programme prevents groin problems among male football players: a cluster-randomised controlled trial. $\mathrm{Br}$ J Sports Med 2019;53:150-7

90 Eckard TG, Padua DA, Hearn DW, et al. The relationship between training load and injury in athletes: a systematic review. Sports Med 2018;48:1929-61. 
91 McKay CD, Steffen K, Romiti M, et al. The effect of coach and player injury knowledge, attitudes and beliefs on adherence to the FIFA 11+ programme in female youth soccer. Br J Sports Med 2014;48:1281-6.

92 Waldén $M$, Hägglund $M$, Werner J, et al. The epidemiology of anterior cruciate ligament injury in football (soccer): a review of the literature from a gender-related perspective. Knee Surg Sports Traumatol Arthrosc 2011;19:3-10.

93 Costello JT, Bieuzen F, Bleakley CM. Where are all the female participants in sports and exercise medicine research? Eur J Sport Sci 2014;14:847-51.

94 Waldén M, Hägglund M, Magnusson H, et al. Anterior cruciate ligament injury in elite football: a prospective three-cohort study. Knee Surg Sports Traumatol Arthrosc 2011;19:11-19.

95 Hägglund M, Waldén M, Ekstrand J. Injuries among male and female elite football players. Scand J Med Sci Sports 2009;19:819-27.

96 Arendt E, Dick R. Knee injury patterns among men and women in collegiate basketball and soccer. NCAA data and review of literature. Am J Sports Med 1995;23:694-701.

97 DiStefano LJ, Dann CL, Chang CJ, et al. The first decade of web-based sports injury surveillance: descriptive epidemiology of injuries in US high school girls' soccer (2005-2006 through 2013-2014) and national collegiate athletic association women's soccer (2004-2005 through 2013-2014). J Athl Train 2018;53:880-92.
98 Hootman JM, Dick R, Agel J. Epidemiology of collegiate injuries for 15 sports: summary and recommendations for injury prevention initiatives. J Ath/ Train 2007; $42: 311-9$

99 Agel J, Rockwood T, Klossner D. Collegiate ACL injury rates across 15 sports: national collegiate athletic association injury surveillance system data update (2004-2005 through 2012-2013). Clin J Sport Med 2016;26:518-23.

100 Montalvo AM, Schneider DK, Yut L, et al. "What's my risk of sustaining an ACL injury while playing sports?" A systematic review with meta-analysis. Br J Sports Med 2019;53:1003-12.

101 Fuller CW, Ekstrand J, Junge A, et al. Consensus statement on injury definitions and data collection procedures in studies of football (soccer) injuries. Br J Sports Med 2006;40:193-201.

102 O'Brien J, Young W, Finch CF. The use and modification of injury prevention exercises by professional youth soccer teams. Scand J Med Sci Sports 2017;27:1337-46.

103 O'Brien J, Young W, Finch CF. The delivery of injury prevention exercise programmes in professional youth soccer: comparison to the FIFA 11. I Sci Med Sport 2017; 20:26-31.

104 Higgins JPT, Green S. Cochrane Handbook for systematic reviews of interventions. version 5.1.0. The Cochrane Collaboration, 2011. 